\title{
Popliteal sciatic nerve block versus spinal anesthesia in hallux valgus surgery
}

\author{
Hyun-Jun Jeon, Young-Chul Park, Jong Nam Lee, and Jun-Seok Bae \\ Department of Anesthesiology and Pain Medicine, St. Mary's Medical Center, Busan, Korea
}

Background: We compared clinical properties and patient satisfaction between spinal anesthesia and popliteal sciatic nerve block (PSNB) for hallux valgus surgery.

Methods: Forty patients undergoing hallux valgus surgery were divided into spinal group (spinal anesthesia with 2.5 $\mathrm{ml}$ of $0.5 \%$ bupivacaine $[\mathrm{n}=20]$ ) and PSNB group (PSNB with $30 \mathrm{ml}$ of $0.75 \%$ ropivacaine mixed with $10 \mathrm{ml}$ of normal saline solution using a nerve stimulator $[\mathrm{n}=20]$ ). The PSNB group used a patient-controlled-analgesia (PCA) pump for postoperative pain control. The quality and side effects were compared between the two groups. A questionnaire was used to evaluate patient satisfaction with the use of anesthetic techniques and postoperative pain control in the PSNB group. This study was assessed 3 days postoperatively by a blinded observer.

Results: Procedure time and time from anesthesia until start of sugery were significantly shorter in the spinal group than those in the PSNB group $(\mathrm{P}<0.01)$. Anesthesia-related complications such as hypotension, bradycardia, shivering, nausea/vomitting, post-dural puncture headache (PDPH) and urinary retension were observed in $15 \%, 10 \%, 5 \%, 5 \%, 10 \%$, and $20 \%$ of patients in the spinal group, respectively. PSNB was not associated with these complications. Patient satisfaction was slightly higher for PSNB than for spinal anesthesia. In the PSNB group, patient satisfaction with postoperative pain-control was $95 \%$ above ordinary satisfaction.

Conclusions: Despite the long duration of the procedure, PSNB is relatively safe, provides an adequate level of anesthesia, effectively controls postoperative pain and reduces side effects. Therefore, PSNB could be a potential anesthetic technique for hallux valgus surgery. (Korean J Anesthesiol 2013; 64: 321-326)

Key Words: Hallux valgus, Nerve block, Popliteal, Sciatic.

Received: June 1, 2012. Revised: 1st, July 13, 2012; 2nd, September 5, 2012; 3rd, September 11, 2012; 4th, September 19, 2012. Accepted: September 22, 2012. Corresponding author: Young-Chul Park, M.D., Department of Anesthesiology and Pain Medicine, St. Mary's Medical Center, Yongho-dong, Nam-gu, Busan 608-090, Korea. Tel: 82-51-932-7114, Fax: 82-51-933-8600, E-mail: pyc9@yahoo.co.kr

(c) This is an open-access article distributed under the terms of the Creative Commons Attribution Non-Commercial License (http:// creativecommons.org/licenses/by-nc/3.0/), which permits unrestricted non-commercial use, distribution, and reproduction in any medium, provided the original work is properly cited. 


\section{Introduction}

Hallux valgus is one of the most common foot deformities. The condition manifests with the hallux presenting with the lateral valgus of the first metatarsophalangeal joint, and the first metatarsal bone is tilted toward the anterior of the second metatarsal bone. The incidence of hallux valgus is highest in females between the ages 40 and 50 [1]. Surgical treatment is performed for patients with severe pain and symptoms. Several anesthetic methods can be chosen, including local anesthesia, nerve block, regional anesthesia, general anesthesia, and, most commonly, spinal anesthesia. Since McCutcheon's [2] report on the use of regional anesthesia for foot surgery in 1965, regional anesthesia has been going through various improvements. In 1992, Myerson et al. [3] reported improved outcomes with anesthesia and found that about $70 \%$ of foot and ankle joint surgeries can be carried out with nerve block. It has been reported that peripheral nerve blocks reduce the cardiovascular side effects associated with regional or general anesthesia $[4,5]$. Among various nerve blocks, popliteal sciatic nerve block (PSNB) has the disadvantage of causing a minor effect on the saphenous nerve territory. However, the area subjected to anesthesia for hallux valgus surgery is mainly affected by the common peroneal nerve territory. In recent years, PSNB has been frequently used for hallux valgus surgery.

In this study, the authors aimed to determine the difference in clinical significance and patient satisfaction between PSNB and spinal anesthesia in prospective hallux valgus surgery.

\section{Materials and Methods}

Patient consent was obtained following authorization from the institutional review board, and 40 patients were selected as the study subjects. The subjects were aged from 20 to 65 years, grade 1 or 2 according to the American Society of Anesthesiologists physical status classification, and due for elective orthopedic surgery. The following patients were excluded from the study: those with spinal malformation, nerve system conditions, regular administration of medication due to medical or surgical disease, hemostatic disorders or infection at the anesthetic injection site. Prior to surgery, an $18 \mathrm{G}$ venous catheter was placed in the left arm to provide fluid during surgery. Prior to administration of anesthesia, a non-invasive blood pressure monitor was placed around the patient's right brachium, and an electrocardiogram and a pulse oximetery were applied continuously. After 5 minutes of rest in the supine position, the blood pressure and heart rate were measured and set as baseline values. The spinal anesthesia group $(n=20)$ was administered with $2.5 \mathrm{ml}$ of $0.5 \%$ hyperbaric bupivacaine (Marcain Spinal 0.5\% Heavy ${ }^{\circledR}$, AstraZeneca, Sweden), and the PSNB group was administered with a mixture of $10 \mathrm{ml}$ of normal saline and $30 \mathrm{ml}$ of $0.75 \%$ ropivacine (Ropiva Inj $7.5 \mathrm{mg} /$ ml, HanLim Pharm, Seoul, Korea).

Spinal puncture was carried out in the spinal anesthesia group. A 26 G Quincke needle (Hakko Co. Ltd, Chikuma, Japan) was used to puncture the location at the central line of the third and fourth lumbar in the lateral decubitus position, and $2.5 \mathrm{ml}$ of $0.5 \%$ hyperbaric bupivacaine was slowly administered after making sure that clear cerebrospinal fluid had been drawn out. The patient was then changed to the supine position, and the sensory block was confirmed through the pin-prick method with a $26 \mathrm{G}$ syringe needle. After confirmation, surgery ensued.

The chosen needle insertion site for the PSNB group was the point $60 \mathrm{~mm}$ upward from the popliteal fossa crease and $10 \mathrm{~mm}$ to the left of the central line while the patient was in the prone position (Fig. 1A). An $18 \mathrm{G} 100 \mathrm{~mm}$ needle coated with an insulator (Contiplex ${ }^{\circledR}$ set, B. Braun, Melsungen, Germany) was used with a nerve stimulator (Digistim 3 plus, Neurotechnology Inc, USA) in the stimulating mode. The popliteal area was disinfected with Betadine and alcohol, and the needle was inserted after preconditioning with local anesthetics. The nerve stimulation mode was activated, followed by connection of
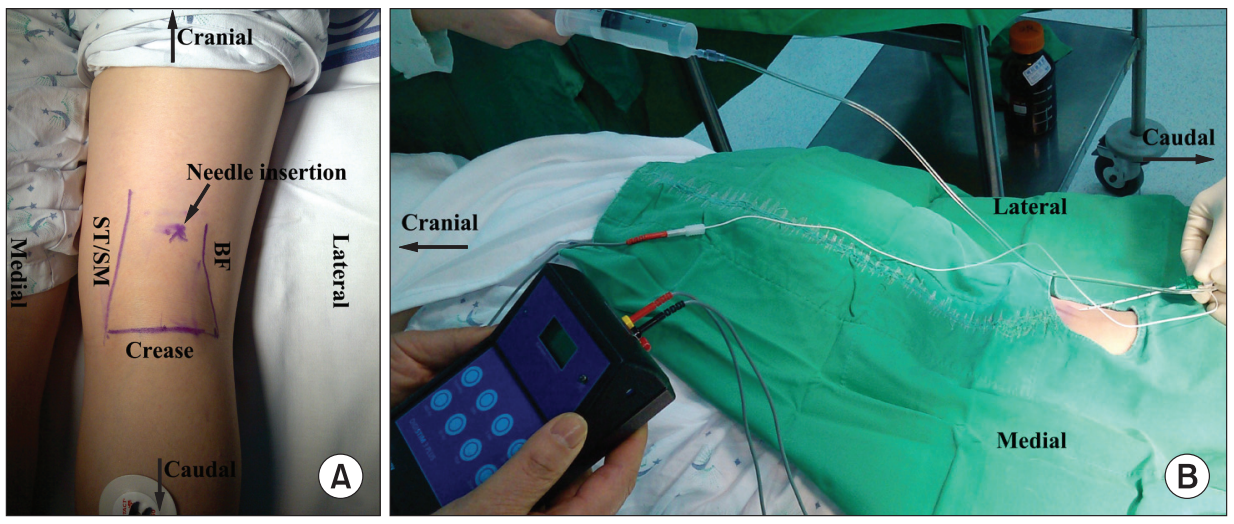

Fig. 1. (A) The needle insertion point is $6 \mathrm{~cm}$ above the popliteal skin crease and $1 \mathrm{~cm}$ lateral to the midline (ST/ SM: semitendinosus muscle/semimembranosus muscle, BF: biceps femoris muscle). (B) The intensity of the stimulating current is initially 1 $\mathrm{mA}$, and then gradually decreases to $0.5 \mathrm{~mA}$. After an appropriate response to nerve stimulation is obtained at a stimulating current of $0.5 \mathrm{~mA}$ or less, $0.5 \%$ ropivacaine is injected. 
the nerve stimulator. Stimulation intensity started at $1.0 \mathrm{~mA}$. While checking foot inversion or dorsiflexion, the intensity was gradually reduced to access the needle around the nerve area. When the muscle maintained minimal contraction under the intensity of $0.5 \mathrm{~mA}$ or less, this value was set as the minimum stimulation intensity (Fig. 1B). The anesthesia syringe was reversed to ensure there was no backflow of blood, and then the anesthetic was slowly injected. A catheter was inserted through the previously inserted needle and moved $3 \mathrm{~cm}$ forward from the end of the needle. The needle was then slowly removed while exercising care to maintain the position of the catheter. A catheter connector and filter were attached, and a sterile bandage was placed at the insertion site on the skin to fix the catheter so that it does not get removed. Sensory loss was confirmed through the pin-prick test, and surgery proceeded after placement of the calf tourniquet.

The duration of the spinal anesthesia procedure was measured from the moment of needle insertion for spinal puncture to completion of administration of the spinal anesthetic. In the PSNB group, the time was measured from the point of local anesthetic infiltration in the skin until fixation of the perineural catheter. Thereafter, the time taken for the block was measured from the moment of administration of spinal or regional anesthesia.

For postoperative pain control, a PCA pump (Accumate 1000, Wooyoung Medical, Seoul, Korea) was connected (basal rate: $10 \mathrm{ml} / \mathrm{h}$, bolus: $2 \mathrm{ml}$, lock out interval: $30 \mathrm{~min}$ ) to administer a mixture of $50 \mathrm{ml}$ mepivacaine (Mevan Inj, HanLim, Seoul, Korea) and $150 \mathrm{ml}$ of normal saline solution. The PCA provided pain block for up to 1 day after surgery, and it was not refilled when all the medication was used.

Extra analgesics and sedatives were administered relative to the degree of the block. About 50-100 $\mu \mathrm{g}$ of fentanyl was administered IV bolus if the patient complained of pain, and 2 mg of midazolam was administered intravenously if the patient complained of anxiety. During surgery, 5-10 mg of ephedrine was administered IV bolus when the systolic blood pressure fell below $100 \mathrm{mmHg}$, and $0.5 \mathrm{mg}$ of atropine was administered intravenously when bradycardia occurred (heart rate $<50$ BPM).

A survey was carried out 3 days post- surgery by a physician

Table 1. Demographic Data

\begin{tabular}{lcc}
\hline & $\begin{array}{c}\text { Spinal group } \\
(\mathrm{n}=20)\end{array}$ & $\begin{array}{c}\text { PSNB group } \\
(\mathrm{n}=20)\end{array}$ \\
\hline Age $(\mathrm{yr})$ & $49.9 \pm 8.5$ & $50.4 \pm 8.1$ \\
Sex $(\mathrm{F} / \mathrm{M})$ & $0 / 20$ & $0 / 20$ \\
Weight $(\mathrm{kg})$ & $58.1 \pm 8.4$ & $56.6 \pm 9.0$ \\
Height $(\mathrm{cm})$ & $155.6 \pm 7.1$ & $158.1 \pm 7.8$ \\
\hline
\end{tabular}

Data are mean \pm SD or number of patient. PSNB: popliteal sciatic nerve block. No significant difference among two groups. who was not involved in this study to allow for objective assessment. The survey questions (yes/no) included unpleasant feeling during surgery, anxiety during surgery, lumbodynia or pain at the popliteal needle insertion site, and pain under the discission. The satisfaction level regarding the anesthesia procedure was surveyed with 5 classifications: very satisfied, satisfied, average, dissatisfied, very dissatisfied. The satisfaction level regarding PCA, which was used in the PSNB group for up to 1 day post-surgery, was also surveyed with 5 classifications: very satisfied, satisfied, average, dissatisfied, very dissatisfied.

The sample size calculation was based on the difference in the incidence of side effects. In the pilot study with 20 patients, the spinal anesthesia group had a total of 3 patients (30\%) who experienced hypotension, nausea and vomiting, and postdural puncture headache (PDPH), respectively. No side effects were reported in the PSNB group. The difference in the incidence of side effects in each group was $30 \%$. The sample size for each group was calculated as 17 patients when the alpha value was 0.05 and the power $80 \%$. When the elimination rate was set up as $20 \%$, the valid sample size was calculated to be 20 patients per group. SPSS version 12 version was used for statistical procedures. Student's t-test and the $\chi 2$ test were used to analysis statistical validation in each group. Statistical significance was defined as a P value of less than 0.05 .

\section{Results}

There were no differences in age, sex, weight, and height between the 2 groups (Table 1 ).

The duration of the procedure, time from anesthesia induction until start of surgery, and surgery time were shorter in the spinal anesthesia group than in the PSNB group $(\mathrm{P}<0.01)$.

Table 2. Anesthetic Data and the Incidence of Adverse Effects

\begin{tabular}{lcc}
\hline & $\begin{array}{c}\text { Spinal group } \\
(\mathrm{n}=20)\end{array}$ & $\begin{array}{c}\text { PSNB group } \\
(\mathrm{n}=20)\end{array}$ \\
\hline Procedure time (min) & $3.5 \pm 1.4$ & $12.8 \pm 5.6^{*}$ \\
Time from block to op. start (min) & $13.4 \pm 3.1$ & $21.8 \pm 8.1^{*}$ \\
Intraoperative medication & & \\
Fentanyl 50 $\mu \mathrm{g}$ & 0 & $2(15 \%)$ \\
$\quad 100 \mu \mathrm{g}$ & 0 & $1(5 \%)$ \\
Midazolam 2 mg & $7(35 \%)$ & $4(20 \%)$ \\
Intraoperative adverse effect & $3(15 \%)$ & 0 \\
Hypotension & $2(10 \%)$ & 0 \\
Bradycardia & $1(5 \%)$ & 0 \\
Shivering & $1(5 \%)$ & 0 \\
Nausea/Vomitting & & 0 \\
Postoperative adverse effect & $2(10 \%)$ & 0 \\
PDPH & $4(20 \%)$ & \\
Urinary retension & & \\
\hline
\end{tabular}

Data are mean \pm SD or number of patient (\%). PSNB: popliteal sciatic nerve block. PDPH: post-dural-puncture hedache $(* \mathrm{P}<0.05)$. 
Table 3. Results of the Study Questionnaire

\begin{tabular}{lcc}
\hline & $\begin{array}{c}\text { Spinal group } \\
(\mathrm{n}=20)\end{array}$ & $\begin{array}{c}\text { PSNB group } \\
(\mathrm{n}=20)\end{array}$ \\
\hline Unpleasant feeling during procedure & $6(30 \%)$ & $5(25 \%)$ \\
Intraoperative anxiety & $6(30 \%)$ & $4(20 \%)$ \\
Pain on needle entry site & $5(25 \%)$ & - \\
$\quad$ Back pain & - & $2(10 \%)$ \\
$\quad$ Pain on popliteal area & 0 & $3(15 \%)$ \\
Incisional pain & & \\
\hline
\end{tabular}

Data are number of patient (\%). PSNB: popliteal sciatic nerve block.

Table 4. Satisfaction with the Anesthetic Technique

\begin{tabular}{lcc}
\hline & $\begin{array}{c}\text { Spinal group } \\
(\mathrm{n}=20)\end{array}$ & $\begin{array}{c}\text { PSNB group } \\
(\mathrm{n}=20)\end{array}$ \\
\hline Very satisfactory & $2(10 \%)$ & $3(15 \%)$ \\
Satisfactory & $7(35 \%)$ & $8(40 \%)$ \\
Ordinary & $3(15 \%)$ & $6(30 \%)$ \\
Unsatisfactory & $3(15 \%)$ & $3(15 \%)$ \\
Very unsatisfactory & $5(25 \%)$ & 0 \\
\hline
\end{tabular}

Data are number of patient (\%). PSNB: popliteal sciatic nerve block.

The anesthesia-related side effects in the spinal anesthesia group were hypotension, bradycardia, shivering, vomiting, postoperative headache, and urinary retension, occurring in $15 \%, 10 \%, 5 \%, 5 \%, 10 \%$, and $20 \%$ of patients, respectively. There was no incidence of side effects in the PSNB group, but it was not statistically significant. Fentanyl, which was used as an extra analgesic, was used in the PSNB group, but not in the spinal anesthesia group. The use of midazolam was more frequent in the spinal anesthesia group than in the PSNB group, but there was no statistical significance (Table 2).

According to the survey, unpleasant feelings during surgery and anxiety were higher in the spinal anesthesia group, but there was no statistical significance. Back pain was present in $25 \%$ (5 cases) of patients in the spinal anesthesia group, and pain at the popliteal needle insertion site occurred in $10 \%$ (2 cases) of patients in the PSNB group. Incisional pain was not reported in the spinal anesthesia group but was reported by $15 \%$ (3 cases) of patients in the PSNB group; however, these figures were not statistically significant (Table 3).

In the satisfaction survey, $60 \%$ (12 cases) of the spinal anesthesia group and $85 \%$ (17 cases) of the PSNB group responded that they were satisfied with the anesthesia procedure (including those who responded 'average'), although the results were not statistically significant (Table 4). The satisfaction survey regarding pain control after the catheter insertion procedure in the sciatic nerve in the PSNB group showed the following results: satisfaction $50 \%$ (10 cases), average $45 \%$ ( 9 cases), and dissatisfaction $5 \%$ ( 1 case) (Table 5).
Table 5. Satisfaction with Postoperative Pain Control in the PSNB Group

\begin{tabular}{lc}
\hline & $\begin{array}{c}\text { PSNB group } \\
(\mathrm{n}=20)\end{array}$ \\
\hline Very satisfactory & 0 \\
Satisfactory & $10(50 \%)$ \\
Ordinary & $9(45 \%)$ \\
Unsatisfactory & $1(5 \%)$ \\
Very unsatisfactory & 0 \\
\hline
\end{tabular}

Data are number of patient (\%). PSNB: popliteal sciatic nerve block.

\section{Discussion}

This study, which was conducted on patients who underwent hallux valgus surgery, resulted in a longer duration for application of anesthesia and longer surgery time in the ropivacaineadministered PSNB group relative to the spinal anesthesia group. However, the PSNB group was associated with a low incidence of anesthesia-related complications, relatively high patient satisfaction with the anesthesia procedure, and effective post-surgical pain control.

The PSNB procedure was first described by Labet in 1923, but it has not been commonly practiced due to limits in the area of application of the block, high incidence rates of side effects including dysesthesia, and problems with the reliability of the block on sciatic nerves or its branches [6]. However, recently, PSNB has been attempted quite often as regional anesthesia for surgeries or post-surgical pain control because its value and reliability are being reassessed in accordance with improvements in insulator needles and nerve stimulators [7].

Moreover, the time taken for the complete block of PSNB has been shortened, and the success rate has been increased due to the use of nerve stimulators or ultrasound. Also, there are several reports with regard to the success rate of PSNB associated with exercise reaction with the use of a nerve stimulator. Sukhani et al. [8] reported that inversion and plantar flexion require a shorter duration to reach complete block than do eversion and dorsiflexion. Taboada et al. [9] reported that plantar flexion reduces the time taken to achieve anesthesia and increases the success rate compared with dorsiflexion, Borgeat et al. [10] reported that inversion and plantar flexion increase the success rate to more than $97 \%$.

There are several ways to perform a sciatic nerve block, such as the front approach, lateral approach, posterior approach, and popliteal approach. In particular, the popliteal approach is the method with which most patients are satisfied because it is easy and the homonymous hamstring muscle can be reserved even after sciatic nerve block, which allows early postsurgical mobilization. In addition, this approach involves less uncomfortable feeling and does not induce pain from the calf tourniquet $[7,11,12]$. Also, a sympathetic nervous system block 
can be expected with PSNB given that the sciatic nerve has sensory nerves and sympathetic nerve fibers in the lower limbs; it can be applicable not only to pain control but also to control post-traumatic foot pain or burning pain [13].

The time taken for complete PSNB varied widely between the patient group. More specifically, 3 patients did not complain of pain when the pin-prick test was done but complained of mild pain under incision, so fentanyl and midazolam were administered. Following this, the surgery proceeded without complaints of pain, and patients did not complain of pain or respiratory disturbance until the surgery was completed. The reason for these differences appears to be related to the dose of regional anesthesia or the time difference between incomplete block and complete block due to the position of the needle during the PSNB procedure. For those patients with incomplete block at the beginning of surgery became a complete block with time. A number of cadaver studies reported that the site where the tibial nerve and the common peroneal nerve branch out from the sciatic nerve at the upper part of popliteal fossa crease is anatomically varied. Vloka et al. [14] reported that branching out was observed $60.0 \pm 27.0$ $\mathrm{mm}$ to the upper part of the popliteal fossa crease, and Saleh et al. [15] reported that the site is somewhere between $50.0 \mathrm{~mm}$ to $180.0 \mathrm{~mm}$. For this reason, delivery of an anesthetic can be limited when regional anesthesia is administered at the inferior site rather than the branching-out site. In some cases, the distance between the tibial nerve and the commom peroneal nerve is several centimeters apart, and the regional anesthesia cannot cover this whole distance. Moreover, the fat layer and connective tissues within the popliteal fossa space interfere with the flow of the anesthetic and delay the time of action. This can induce individual time differences to the complete block or cause incomplete block. Therefore, many methods, such as the double-injection technique, $[16,17]$ are used to identify the tibial nerve and the commom peroneal nerve separately and administer at each point to increase the success rate of PSNB. The needle should be positioned $10 \mathrm{~cm}$ above the popliteal fossa crease where the sciatic nerve branches out, as was reported by Paqueron et al. [17], but these theories require verification through clinical studies involving large numbers of patients.

Peripheral nerve block can limit the side effects of general anesthesia, such as nausea and vomiting or cardiovascular adverse reactions. Also, it is known to reduce several side effects induced by regional anesthesia. In the present study, there was no incidence of hypotension, bradycardia, shivering, postoperative urinary retention, and PDPH relative to spinal anesthesia. In addition, surgery could be carried out in hemodynamically unstable patients who were on on anticoagulation therapy without facing a huge risk. It can also be an appropriate anesthetic procedure in geriatric patients who have anatomical issues due to degenerative changes [18]. The incidence of PDPH is higher in females than males and among younger patients [19], which was in accordance with this study showing higher incidence in female patients in their 30s and 50s. In addition, hallux valgus generally occurs in females, which encompasses the high-risk age group for PDPH.

Even with the above advantages, the side effects of peripheral nerve block surgery limit the usefulness of the procedure. The common side effects are: incomplete block, direct nerve damage, localized hematoma and consequent ischemic damage, infection, and the risk of intravenous administration of local anesthetic [18]. There were no side effects in the PSNB group in this study, but Hajek et al. [20] reported superficial peroneal nerve and sural nerve damage in 3 patients $(1.91 \%)$ out of 157 patients who were treated with continuous peripheral nerve block (CPNB) at the popliteal. Possible causes were exposed peroneal nerve and sural nerve damage, nerve toxicity of the local anesthesia, direct nerve damage and ischemia, and blockage of the local anesthetic flow in relation to the tourniquet. Despite the small chance, patients should be informed about such side effects. Those who require an appropriate anesthetic procedure need close monitoring for the incidence of side effects, and more investigation is warranted to decide on the ideal anesthetic procedure.

In the patient satisfaction survey, PSNB was shown to have relatively high satisfaction compared with spinal anesthesia. This can be explained by the incidence of back pain from spinal anesthesia and various side effects during and after surgery, as these played important roles in reducing patients' satisfaction. Aside from 2 patients who developed PDPH, 5 patients (25\%) indicated they were "very dissatisfied" due to back pain. In the PSNB group, satisfaction was low among patients who experienced pain at the needle insertion site, a longer procedural time, and pain under discission. These differences in the satisfaction levels would be greater if comparisons were made with a $26 \mathrm{G}$ needle, a thicker needle, in the spinal anesthesia group or with a higher amount of spinal anesthesia.

The satisfaction survey about postoperative pain control after sciatic nerve catheterization could not be used for the comparative study because patients with the spinal anesthetic rejected the use of intravenous patient-controlled analgesia (IV PCA) or the use of various methods of post-surgical pain control. However, in PSNB patients who used a PCA due to catheter insertion around the sciatic nerve, there was no incidence of side effects such as nausea and vomiting, which were common in those who recieved IV PCA or oral administration. Furthermore, 95\% of PSNB patients showed satisfaction, including "average" satisfaction. Elliot et al. [21] reported that continuous nerve block due to sciatic nerve catheterization after foot and ankle 
joint surgery can reduce the need for extra pain relief and pain after surgery. Navas et al. [22] reported that this procedure increases pain relief after surgery, thus improving patient satisfaction.

Although the study was carried out prospectively, the limitation of the study was that there was no random sampling and no blinding, and the comparisons of satisfaction scores and the levels of pain relief were not done in a double-blinded fashion. To validate the significance of the difference in side effects and patient satisfaction, a further study with a larger patient population is warranted.

In summary, this study demonstrated that in hallux valgus surgery patients, the time taken for the procedure and nerve block in the PSNB group with $0.5 \%$ ropivacaine administration was longer than that taken for the spinal anesthesia group with $2.5 \mathrm{ml}$ of $0.5 \%$ bupivacaine administration. There were individual variations in the time to complete the nerve block, but PSNB was determined as relatively safe, provided an appropriate level of anesthesia, reduced possible side effects from the spinal anesthesia, and showed excellent results on postoperative pain control. The authors suggest that PSNB be considered as the anesthetic procedure for hallux valgus surgery anesthesia and for postoperative pain control, especially in patients for whom spinal anesthesia is not feasible.

\section{References}

1. Ahn YJ, Hahn SH, Yang BK, Yi SR, Yoo JH, Chung BJ, et al. The results of distal chevron osteotomy for hallux valgus in young adult. J Korean Foot Ankle Soc 2005; 9: 158-61.

2. McCutcheon R. Regional anaesthesia for the foot. Can Anaesth Soc J 1965; 12: 465-74.

3. Myerson MS, Ruland CM, Allon SM. Regional anesthesia for foot and ankle surgery. Foot Ankle 1992; 13: 282-8.

4. Auroy Y, Narchi P, Messiah A, Litt L, Rouvier B, Samii K. Serious complications related to regional anesthesia: results of a prospective survey in France. Anesthesiology 1997; 87: 479-86.

5. Yazigi A, Madi-Gebara S, Haddad F, Hayeck G, Tabet G. Intraoperative myocardial ischemia in peripheral vascular surgery: general anesthesia vs combined sciatic and femoral nerve blocks. J Clin Anesth 2005; 17: 499-503.

6. Rorie DK, Byer DE, Nelson DO, Sittipong R, Johnson KA. Assessment of block of the sciatic nerve in the popliteal fossa. Anesth Analg 1980; 59: 371-6.
7. Singelyn FJ, Gouverneur JM, Gribomont BF. Popliteal sciatic nerve block aided by a nerve stimulator: a reliable technique for foot and ankle surgery. Reg Anesth 1991; 16: 278-81.

8. Sukhani R, Nader A, Candido KD, Doty R Jr, Benzon HT, Yaghmour E, et al. Nerve stimulator-assisted evoked motor response predicts the latency and success of a single-injection sciatic block. Anesth Analg 2004; 99: 584-8.

9. Taboada M, Atanassoff PG, Rodríguez J, Cortés J, Del Rio S, Lagunilla J, et al. Plantar flexion seems more reliable than dorsiflexion with Labat's sciatic nerve block: a prospective, randomized comparison. Anesth Analg 2005; 100: 250-4.

10. Borgeat A, Blumenthal S, Lambert M, Theodorou P, Vienne P. The feasibility and complications of the continuous popliteal nerve block: a 1001-case survey. Anesth Analg 2006; 103: 229-33.

11. Kilpatrick AW, Coventry DM, Todd JG. A comparison of two approaches to sciatic nerve block. Anaesthesia 1992; 47: 155-7.

12. Zetlaoui PJ, Bouaziz H. Lateral approach to the sciatic nerve in the popliteal fossa. Anesth Analg 1998; 87: 79-82.

13. Loeser JD. Bonica's management of pain. 3rd ed. Philadelpia, Lipincott, Williams \& Wilkins. 2001, pp 1890-952.

14. Vloka JD, Hadzic A, April E, Thys DM. The division of the sciatic nerve in the popliteal fossa: anatomical implications for popliteal nerve blockade. Anesth Analg 2001; 92: 215-7.

15. Saleh HA, El-fark MM, Abdel-Hamid GA. Anatomical variation of sciatic nerve division in the popliteal fossa and its implication in popliteal nerve blockade. Folia Morphol (Warsz) 2009; 68: 256-9.

16. Bailey SL, Parkinson SK, Little WL, Simmerman SR. Sciatic nerve block. A comparison of single versus double injection technique. Reg Anesth 1994; 19: 9-13.

17. Paqueron X, Bouaziz H, Macalou D, Labaille T, Merle M, Laxenaire MC, et al. The lateral approach to the sciatic nerve at the popliteal fossa: one or two injections? Anesth Analg 1999; 89: 1221-5.

18. Jeon YT. Peripheral nerve block for anesthesia in patients having knee arthroplasty. Korean J Anesthesiol 2012; 62: 403-4.

19. Rasmussen BS, Blom L, Hansen P, Mikkelsen SS. Postspinal headache in young and elderly patients. Two randomised, double-blind studies that compare 20-and 25-gauge needles. Anaesthesia 1989; 44: 571-3.

20. Hajek V, Dussart C, Klack F, Lamy A, Martinez JY, Lainé P, et al. Neuropathic complications after 157 procedures of continuous popliteal nerve block for hallux valgus surgery. A retrospective study. Orthop Traumatol Surg Res 2012; 98: 327-33.

21. Elliot R, Pearce CJ, Seifert C, Calder JD. Continuous infusion versus single bolus popliteal block following major ankle and hindfoot surgery: a prospective, randomized trial. Foot Ankle Int 2010; 31: 1043-7.

22. Navas AM, Gutierrez TV, Moreno ME. Continuous peripheral nerve blockade in lower extremity surgery. Acta Anaesthesiol Scand 2005; 49: 1048-55 